\title{
Production of time intervals from segmented and nonsegmented inputs
}

\author{
SIMON GRONDIN \\ Université Laurentienne, Sudbury, Ontario, Canada
}

\begin{abstract}
One important factor influencing the accuracy of a timing estimate is the counting activity that a human subject may adopt. In the present study, the usefulness of this activity is evaluated with a strategy whereby subjects are presented segmented and nonsegmented intervals, before they start to produce a series of these intervals, using a finger-tapping procedure. The results are mainly analyzed in the light of Killeen and Weiss's (1987) model, which addressed this question of counting. The results revealed that (1) a scalar property gives a better description of the pacemaker activity than does a Poisson process, and (2) an optimal timing performance would be reached with the utilization of subintervals with an approximate value of $400 \mathrm{msec}$. Finally, the discussion also incorporates an analysis of the variability related to the motor component in a tapping task.
\end{abstract}

Most people who are required to estimate an interval of time will spontaneously adopt a strategy for increasing the reliability of their estimates. This strategy will be to break a larger interval into smaller ones. To explain the usefulness of this strategy, Killeen and Weiss (1987) reported a general theoretical framework. Indeed, their analysis holds for empirical reports, whether or not they involve explicit counting.

In brief, the component analysis of Killeen and Weiss (1987) states that given the average duration of an entire interval $t$ (or $\mu_{\mathrm{T}}$ ), the average number of subintervals $n$ (or $\mu_{\mathrm{N}}$ ), and the average duration of a subinterval $d$ (or $\left.\mu_{\mathrm{D}}\right)$,

$$
t=n d,
$$

and

$$
\sigma_{\mathrm{T}}^{2}=n \sigma_{\mathrm{D}}^{2}+d^{2} \sigma_{\mathrm{N}}^{2}
$$

The total variance is then a combination of errors coming from the weighted sum of two sources, $\sigma_{\mathrm{D}}^{2}$ and $\sigma_{\mathrm{N}}^{2}$. The growth of these variances is assumed to correspond to these equations:

$$
\sigma_{\mathrm{D}}^{2}=\alpha_{2} d^{2}+\alpha_{1} d+\alpha_{0}
$$

and

This research was supported by a grant from the Natural Sciences and Engineering Research Council of Canada, and by a Summer Employment/Experience Development program from Employment and Immigration Canada. I wish to thank Lester Krueger, Peter Killeen, and two anonymous reviewers for their valuable comments on earlier versions of this article. Special thanks to Stan Koren for his excellent technical assistance, Lynn Metthe for her help in the data collection, and Geoffrey Tesson for his help with the English language. This study was presented at the 1992 convention of the Canadian Psychological Association, held in Québec City in June. Correspondence concerning this paper should be addressed to Simon Grondin, Department of Psychology, Laurentian University, Ramsey Lake Road, Sudbury, Ontario, Canada P3E 2C6 (e-mail: sgrondin@nickel.laurentian.ca).

$$
\sigma_{\mathrm{N}}^{2}=\beta_{2} n^{2}+\beta_{1} n+\beta_{0} .
$$

Killeen and Weiss referred to Equations 3 and 4 as the fundamental error equations.

In spite of the relevance of the question, the literature has reported very little empirical evidence that permits the evaluation of the effect of explicit counting or the benefits of a segmentation strategy when one is estimating time (Getty, 1976; Gilliland \& Martin, 1940; Hicks \& Allen, 1979; Petrusic, 1984). A recent series of investigations by Fetterman and Killeen (1990) provides more information about the optimal tradeoff between $n$ and $d$ when one is estimating $t$. In their experiments, they asked the subjects to reproduce intervals after having been presented tone durations. These reproductions of intervals involved a series of taps on a key until the target duration was reached; the number of responses $(n)$, the duration between successive taps $(d)$, and the total duration of their reproductions $(t)$ were recorded. They mainly reported that although the rate of responding - that is, the length of $d$-differed considerably between subjects, it remained approximately the same for one given subject for different values of $t$; the variability in counting $(n)$ was mainly responsible for explaining the total variability of estimates; there were less counting errors when subjects used explicit counting; and the variability increased when subjects were asked to deviate from their preferred rates of responding.

The series of experiments by Fetterman and Killeen (1990) was a major attempt to test Killeen and Weiss's (1987) analysis, but some important questions remained open or have arisen following these experiments. One question regards the optimal tradeoff between $n$ and $d$ for getting the best timing performance. Another key question still requiring experimental work concerns the properties of emission of pulses by the pacemaker in a pacemaker-counter (or clock-counter) system. Indeed, most models of time estimation attribute the variability 
of judgments to a clock that would follow some form of Weber's law (Getty, 1975; Treisman, 1963) or to a clockcounter device in which it is assumed that the counting component is without error and that the clock has properties of a Poisson system (Creelman, 1962; Rousseau, Picard, \& Pitre, 1984). On the other hand, Kristofferson (1977) has developed a model in which it is assumed that the clock is not a source of error. The animal literature also shows divergent points of view, since Gibbon and Church (1984) assume a scalar property, whereas Killeen and Fetterman (1988) assume the clock to have the characteristics of the Poisson process. Note that recent findings with bisection by humans seem to support the scalar property described in the animal literature (Allan \& Gibbon, 1991; see Wearden, 1991a, 1991b for discussion on this issue). Finally, recent studies by Fetterman and Killeen (1992) have shown that a generalized form of Weber's law provides a good description of time discrimination by humans and by animals (pigeons).

In the following empirical study, one strategy was introduced that provided further information about the properties of the pacemaker and about the optimal timing. With this strategy, the variability of time judgments was calculated on the basis of the differences between the length of intervals created by a series of finger taps. Before these interval productions, some models of the target interval are presented to the subject, and, according to the trials, these models are not divided or are divided by two, three, or four. In terms of Equation 1, the number of subintervals $n$ for a given trial is fixed. Thus, $n$ always being small, the counting errors that would result from Equation 4 are here assumed to equal 0 . Then, the observed variability in these cases is assumed to be due to the variance of the subintervals (Equation 3). The experiment is designed to provide different tests, by having different conditions of $n$, for evaluating the increment of the variability due to $d$ as a function of $t$. Also, the subjects are asked to produce two different series of intervals-either four or eight intervals (five or nine taps)-after having been presented the inputs (i.e., the models of the target interval). This is expected to provide an opportunity for the segmentation effects to be tested by two sets of evaluations of the variability of productions.

\section{METHOD}

\section{Subjects}

Sixteen volunteers, from 18 to 24 years old, participated in this experiment. They were paid $\$ 16$ for their participation.
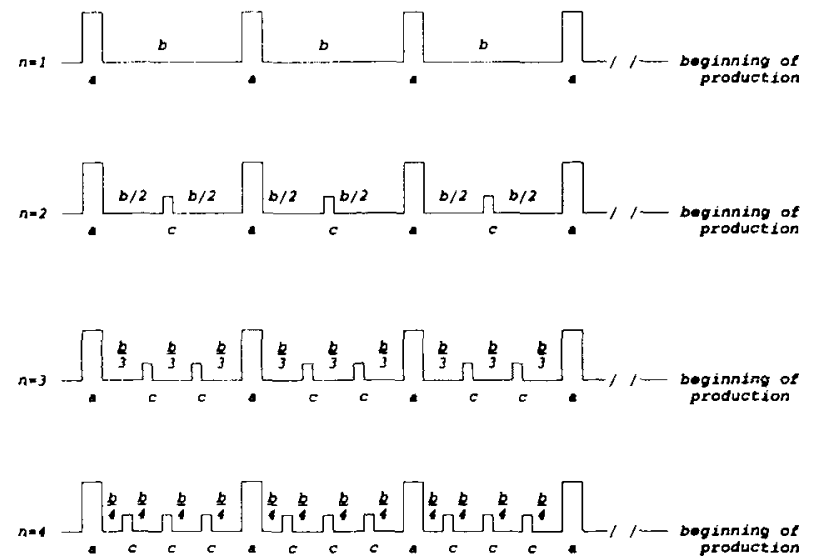

Figure 1. Auditory sequences presented before the subject begins to produce intervals. (a) A 15 -msec, 5-kHz tone marking (b) the interval of $1.2,2.4,3.6$, or $4.8 \mathrm{sec}$, which either is not subdivided $(n=1)$ or (c) is subdivided by two $(n=2)$, three $(n=3)$, or four $(n=4)$ dividers, each of which consists of a 15-msec, 1-kHz tone.

\section{Apparatus and Stimuli}

The experiment was run under the control of a Zenith microcomputer, which was linked to a response box that contained the pushbutton used to produce the intervals. The auditory markers defining the interval that the subject had to produce were presented binaurally through a headphone. Each marker was a $15-\mathrm{msec}$ and $5-\mathrm{kHz}$ tone recorded at $70 \mathrm{~dB}$ for each ear. Each sound dividing the standard interval was a $15-\mathrm{msec}$ and $1-\mathrm{kHz}$ tone with a $70-\mathrm{dB}$ intensity.

\section{Procedure}

Each trial started with the presentation of a series of four successive auditory markers defining three examples of the target intervals to be produced. The task of the subject was then to produce a series of taps separated by a duration equal to the target interval. According to the trials, the subject had to produce four intervals (tapping five times) or eight intervals (tapping nine times). The target interval was interrupted by zero, one, two, or three dividers, the duration in the three latter cases being respectively subdivided into $n=2,3$, or 4 equal subintervals (Figure 1). There were four conditions of target duration $(t): 1.2-, 2.4-, 3.6-$, and 4.8-sec intervals. There were four sessions, one for each duration to produce. The order of presenting of the four duration conditions was balanced according to a Latin square (see Table 1).

Each session was divided into six identical cycles of eight trials. Each cycle was separated by a 20 -sec pause, prolonged according to the wish of the subject. The eight trials of a cycle were separated by an 8 -sec pause, a 500 -msec warning signal, and a $2-\mathrm{sec}$ pause. The order of presenting the divider conditions was balanced according to a Latin square (Table 1); indeed, for this, the trials were grouped in four blocks. Trials $1-2,3-4,5-6$, and $7-8$ were pooled together. Each block corresponded to a divider condition.

Table 1

Counterbalancing of the Order of Passage of the Target Durations from Session to Session and of the Number of Dividers from Trial to Trial

\begin{tabular}{|c|c|c|c|c|c|c|c|c|}
\hline \multirow[b]{2}{*}{ Sequence } & \multicolumn{4}{|c|}{ Target Duration (Seconds) } & \multicolumn{4}{|c|}{ No. Dividers } \\
\hline & Session 1 & Session 2 & Session 3 & Session 4 & Trials 1-2 & Trials 3-4 & Trials 5-6 & Trials 7.8 \\
\hline 1 & 2.4 & 3.6 & 1.2 & 4.8 & 2 & 3 & 1 & 0 \\
\hline 2 & 3.6 & 4.8 & 2.4 & 1.2 & 3 & 0 & 2 & 1 \\
\hline 3 & 1.2 & 2.4 & 4.8 & 3.6 & 1 & 2 & 0 & 3 \\
\hline 4 & 4.8 & 1.2 & 3.6 & 2.4 & 0 & 1 & 3 & 2 \\
\hline
\end{tabular}


Thus, having four different orders for each of the divider conditions and the duration conditions meant that each of the 16 subjects was subjected to a different order. Of these 16 different orders, 8 required the subject to produce the four-interval series (five taps) during Blocks $1,3,5$, and 7 , and the eight-interval series (nine taps) during Blocks 2, 4, 6, and 8; and the other 8 orders required the subjects to produce the eight-interval series during Blocks 1 , 3,5 , and 7 , and the four-interval series during Blocks 2, 4, 6, and 8 .

Familiarization trials were carried out at the beginning of the first session. In one block, the subject had to tap 5 times after having been presented four auditory stimuli separated by a 1-sec interval. In a second block, the subject had to tap 16 times after having been presented three 2-sec intervals (four auditory stimuli) segmented into two equal parts by a divider. This was to familiarize the subject with the pushbutton and the auditory stimuli marking the intervals and the dividers.

The sessions with the 1.2-, 2.4-, 3.6-, and 4.8-sec productions lasted, respectively, about $25,35,45$, and $55 \mathrm{~min}$. The subject was asked not to use the subdivision of the preceding block; this instruction was useful for blocks in which the intervals were not subdivided. Finally, for each case of the target interval $\times$ divider condition $\times$ number of taps combinations $(4 \times 4 \times 2)$, there were six evaluations. For the analysis of the coefficient of variation, the mean of these six evaluations was calculated.

\section{RESULTS}

Although variability is the dependent variable that will provide answers to the questions justifying this study, the mean productions are also reported in the next paragraph.

\section{Mean Production}

The mean productions in each experimental condition of this experiment are reported in Table 2. The productions for each of the segmentation conditions and of the four- or eight-interval production conditions were compared. For each duration condition, a randomized block factorial analysis of variance $(2 \times 4$; Kirk, 1982$)$ was conducted. With the 1.2-sec target duration, the length of production varied with segmentation $[F(3,105)=15.04$, $p<.01]$, but the other main effect and the interaction were not significant. The Tukey test revealed that having two or three dividers leads to longer production than does having zero or one divider. In the 2.4-sec condition, only the segmentation conditions showed significant differences $[F(3,105)=3.55, p<.05]$. The Tukey test did not reveal any significant differences; we may assume at least that having no divider leads to longer productions than does having one divider. With the 3.6-sec condition, once again only the segmentation condition showed significant differences $[F(3,105)=14.44, p<.01]$. Having no divider gave longer productions than did having one or two dividers, and with three dividers, the productions were longer than with one divider. Finally, with the 4.8sec condition, only the segmentation condition gave significant differences $[F(3,105)=17.77, p<.01]$. Having no divider provoked the production of longer intervals than did having one, two, or three dividers.

\section{Variability as a Function of $t$}

In this section of the analysis of the results, the focus is on the increases in the variability of a series of intervals produced by finger taps as a function of the length of the interval, $t$, to be produced. The variability is measured by using two indices, the standard deviation and the variance, and the purpose of the analysis is to see how these indices increased in accordance with $t$. This analysis was done for each subject for each of eight conditions (4 segmentation conditions $\times 2$ number of taps conditions). For each of these cases, a linear regression analysis was performed for evaluating the best-fitting straight line for describing the increases in each standard deviation and variance with increments in $t$. For each subject, regression analyses were performed, in which were included the six trials for each target duration (24 points). The value of $t$ introduced in the analysis was the value of the production and not the value of the target.

The best predictions, as expressed by $R^{2}$, were generally observed when the standard deviation was plotted with t. This superiority of standard deviation for predicting variability with time was observed both with the condition in which no segmented input was presented and with conditions with two, three, and four subintervals. In only 5 of the 128 individual regression analyses (4 segmentation conditions $\times 2$ number of taps conditions $\times 16$ subjects) was the $R^{2}$ superior with the variance. Also, in 11 of the 128 evaluations did the $R^{2}$ fail to reach the .05 critical level with the variance, and in only 4 did it fail to do so with the standard deviation. The average $R^{2}$ results for the four- and eight-interval production conditions were, respectively, .414 (.307) and .543 (.423), .526 (.370) and .664 (.520), .450 (.341) and .615 (.462), and $.390(.321)$ and $.506(.493)$ for each $n=1,2,3$, and 4 segmentation conditions (the values in parentheses are those calculated with the variance, and the other values were obtained with the standard deviation).

On the other hand, a linear regression analysis was performed, with the standard deviation and with the variance, for each of the eight- conditions, on the basis of the scores of the 16 subjects. These analyses are reported in Table 3 . It shows that for group data, as well as for individual data,

Table 2

Mean Production (in Milliseconds) in Each Condition; Group Results

\begin{tabular}{|c|c|c|c|c|c|c|c|c|c|c|c|c|c|c|c|c|}
\hline \multirow{3}{*}{$\begin{array}{l}\text { No. Intervals } \\
\text { in Series }\end{array}$} & \multicolumn{16}{|c|}{ No. Dividers } \\
\hline & \multicolumn{4}{|c|}{$\begin{array}{c}\text { 1.2-Sec Target } \\
\text { Interval }\end{array}$} & \multicolumn{4}{|c|}{$\begin{array}{l}\text { 2.4-Sec Target } \\
\text { Interval }\end{array}$} & \multicolumn{4}{|c|}{$\begin{array}{l}\text { 3.6-Sec Target } \\
\text { Interval }\end{array}$} & \multicolumn{4}{|c|}{$\begin{array}{c}\text { 4.8-Sec Target } \\
\text { Interval }\end{array}$} \\
\hline & 0 & 1 & 2 & 3 & 0 & 1 & 2 & 3 & 0 & 1 & 2 & 3 & 0 & 1 & 2 & 3 \\
\hline $\begin{array}{l}4 \\
8\end{array}$ & $\begin{array}{l}1,322 \\
1,320\end{array}$ & $\begin{array}{l}1,310 \\
1,324\end{array}$ & $\begin{array}{l}1,369 \\
1,383\end{array}$ & $\begin{array}{l}1,390 \\
1,388\end{array}$ & $\begin{array}{l}2,567 \\
2,575\end{array}$ & $\begin{array}{l}2,497 \\
2,487\end{array}$ & $\begin{array}{l}2,516 \\
2,514\end{array}$ & $\begin{array}{l}2,552 \\
2,579\end{array}$ & $\begin{array}{l}3,909 \\
3,828\end{array}$ & $\begin{array}{l}3,469 \\
3,466\end{array}$ & $\begin{array}{l}3,610 \\
3,605\end{array}$ & $\begin{array}{l}3,717 \\
3,708\end{array}$ & $\begin{array}{l}5,034 \\
5,101\end{array}$ & $\begin{array}{l}4,695 \\
4,659\end{array}$ & $\begin{array}{l}4,556 \\
4,627\end{array}$ & $\begin{array}{l}4,804 \\
4,782\end{array}$ \\
\hline
\end{tabular}


Table 3

Regression Equation and $\mathbf{R}^{2}$ in Each Condition; Group Results

\begin{tabular}{|c|c|c|c|c|c|}
\hline \multirow{2}{*}{$\begin{array}{l}\text { Divider } \\
\text { Condition }\end{array}$} & \multirow{2}{*}{$\begin{array}{l}\text { No. Intervals } \\
\text { in Series }\end{array}$} & \multicolumn{2}{|c|}{ Standard Deviation } & \multicolumn{2}{|l|}{ Variance } \\
\hline & & Regression Equation & $R^{2}$ & Regression Equation & $R^{2}$ \\
\hline 0 & 4 & $.0556 x-22.15$ & .3856 & $25.91 x-42,186$ & .2905 \\
\hline 0 & 8 & $.0673 x-26.72$ & .4774 & $35.53 x-58,574$ & .3314 \\
\hline 1 & 4 & $.0648 x-44.38$ & .4550 & $27.26 x-43,944$ & .2713 \\
\hline 1 & 8 & $.0759 x-49.88$ & .5947 & $36.37 x-61,058$ & .4180 \\
\hline 2 & 4 & $.0497 x-22.66$ & .3766 & $17.99 x-27,786$ & .2401 \\
\hline 2 & 8 & $.0614 x-33.02$ & .4838 & $24.51 x-38,735$ & .3213 \\
\hline 3 & 4 & $.0337 x+8.48$ & .3006 & $10.59 x-13,386$ & .1857 \\
\hline 3 & 8 & $.0488 x-4.58$ & .4263 & $19.05 x-27,776$ & .2899 \\
\hline
\end{tabular}

Note-For the regression equation, $t$ is on the $x$-axis, and standard deviation or variance is on the $y$-axis.

the standard deviation provided a better account than did the variance for the increases in the variability of interval productions as a function of $t$. Also, as for most cases of the individual analyses, the $R^{2}$ values were superior with the eight- as opposed to the 4-interval production condition. Finally, the intercept was always close to zero when the analysis was done with the standard deviation. However, with the variance, the intercept was far from zero, which is difficult to interpret. This result with the intercept also suggests that the standard deviation, in comparison with the variance, gave a better description of the increases in variability as a function of $t$.

\section{Coefficient of Variation}

In this part of the analysis, the purpose was to compare the performances under different conditions when the different target durations were transformed within a comparable form. To accomplish this, the index of performance was a coefficient of variation, with the variability-expressed with the standard deviation-divided by duration produced by the subject. In each condition, the dependent variable was a mean of six trials.

A general illustration of the results is provided by Figure 2 . It shows that the variability generally decreased

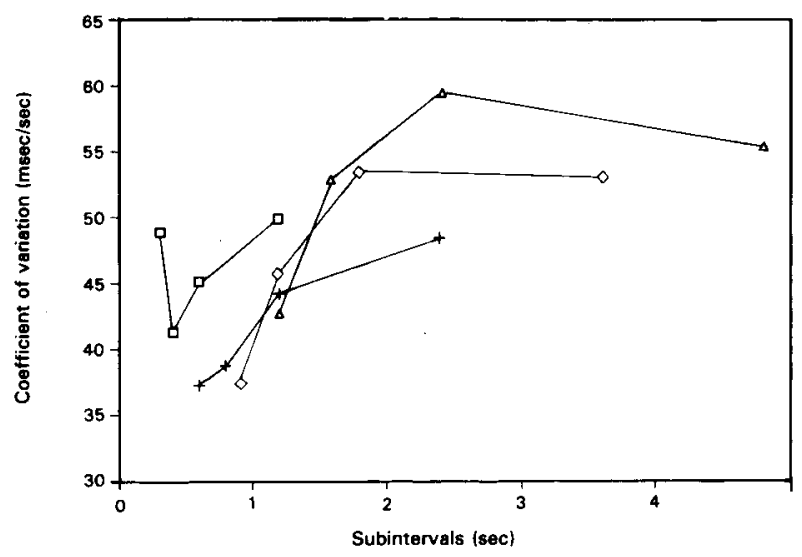

Figure 2. Coefficient of variation for each condition. The points are placed as a function of the length of the subintervals. (Open squares, 1.2-sec production; plus signs, 2.4-sec production; open diamonds, 3.6-sec production; open triangles, 4.8-sec production.) with increments in $n$. Two facts contradict this tendency. There were no benefits at 4.8 and $3.6 \mathrm{sec}$, in comparison with the no-segmentation condition, in which there was only one divider; and at $1.2 \mathrm{sec}$, performance decreased when the subintervals increased from three to four.

These results of the coefficient of variation were submitted to a statistical analysis following a randomized block factorial design ( 2 numbers of taps $\times 4$ segmentations $\times 4$ durations). There were significant differences between the four- and eight-interval productions $[F(1,465)$ $=74.59, p<.01]$ : the variability of the productions increased with number of intervals to be produced following the auditory input. There were also significant differences among the segmentation conditions $[F(3,465)=$ $20.08, p<.01]$ and among the duration conditions $[F(3,465)=16.45, p<.01]$. Of all the interactions, only that of segmentation $\times$ duration was significant $[F(9,465)$ $=4.27, p<.01]$. For the analysis of this interaction, the average of productions in the four- and eight-interval productions was computed. All four tests on simple main effects for the duration revealed significant differences. The Tukey test revealed the following significant differences $(p<.05)$. At $1.2 \mathrm{sec}$, productions were more variable with no divider than with two; at 2.4-sec, productions were more variable with no divider than with two or three, and more variable with one than with three dividers; at $3.6 \mathrm{sec}$, the use of zero or one divider showed greater variability than did that of three dividers; and at $4.8 \mathrm{sec}$, having three dividers permitted lower variability than did having three, one, or two dividers.

Finally, another angle of analysis deserves attention. Having to produce the 1.2-sec intervals with no segmentation, the 2.4-sec intervals with two subintervals, the 3.6sec intervals with three subintervals, and the 4.8-sec intervals with four subintervals all resulted in equal 1.2sec internal subintervals. These four conditions were compared and analyzed with a randomized block factorial analysis of variance ( 2 interval sequences $\times 4$ ), which showed a significant difference between the four- and eightinterval productions $[F(1,105)=28.79, p<.01]$; the variability was greater with the eight-interval productions. Significant differences among the duration conditions were found $[F(3,105)=3.00, p<.05]$ and the interaction was not significant. A Tukey test revealed that the 4.8 - 
sec condition with three dividers led to less variability than did the 1.2-sec condition. This result is illustrated in Figure 2.

\section{DISCUSSION}

The main purpose of this study was to compare how the standard deviation and the variance of a series of intervals produced by finger tapping increased with $t$. The comparison was made in different conditions of segmentation in which the number of subintervals, $n$, was kept small. Thus, the observed variability could be attributed to the timing component of Killeen and Weiss's (1987) model. Although the results obtained with the variance could provide quite a good account for the data, a better account was provided by the standard deviation. This observation has the following theoretical significance. It shows that the timing could be assumed to be scalar (i.e., it is Weber-like), a finding consistent with those of Getty (1976) or Gibbon and Church (1984). Thus, in this study, the data would rule out the possibility that the timing component is Poisson-like. Such a conclusion has not been supported by other studies (Creelman, 1962; Wing and Kristofferson, 1973) or by the recent analysis of Fetterman \& Killeen (1990). Note that some data reported to support a Poisson model for the pacemaker could lead to unclear conclusions after reanalysis-see, for example, Ivry and Corcos (in press), who reanalyzed Wing and Kristofferson's (1973) results and showed that the standard deviation provided as good an account as did the variance for describing the increments in variability with $t$. In brief, in terms of Equation 3 of the Killeen and Weiss model, the data of the present study suggested for the timing component that $\alpha_{2}>0$ and $\alpha_{1}=0$, since the increment of variability with $t$ was described better with the standard deviation than with the variance. This result is consistent with a scalar description of timing by humans (Allan \& Gibbon, 1991; Wearden, 1991a).

One very important question following Killeen and Weiss's (1987) model concerns the value of subintervals, $d$, for optimizing the timing accuracy. Fetterman and Killeen (1990) reported that optimal timing-that is, minimization of the variability of $t$ estimates - would be obtained with values of $d$ in the range of a quarter to a half of a second. The results of the present experiment are in agreement with this conclusion. Indeed, in direct relation to optimal timing, two aspects of these data deserve attention: 1) The analysis of the variability based on the coefficient of variation generally showed a clear benefit from reducing the length of the subintervals, but this advantage only became evident when $d$ was smaller than $1.8 \mathrm{sec}$; in both the 3.6- and the 4.8-sec target duration conditions, dividing $t$ into two subintervals did not lead to any improvement in the performance. In other words, not only might there be an optimal value for $d$, but past a given point, subintervals could be too large to provide help in timing. (2) It is not to the advantage of the subject to count as fast as possible. In the 1.2-sec target duration condition, performance deteriorated when passing from three to four subintervals. On the basis of these group data, $d=300 \mathrm{msec}$ would be too short to provide the best performance: an approximate value of $d=$ $400 \mathrm{msec}$ would permit an optimal timing. Fetterman and Killeen (1990) have also observed, in an experiment in which the subjects were asked to increase their rates of counts, that the subjects should not count as fast as possible. This result of Fetterman and Killeen was found in a context in which $\alpha_{0}$ was estimated to be about zero. In such a case, the model of Killeen and Weiss (1987) predicted that $d$ should be as small as possible. Fetterman and Killeen (1990) concluded from this analysis that the pacemaker and the counter might not be independent component processes, which is contrary to a basic assumption of the Killeen and Weiss (1987) model. An example of nonindependence of the two components, as noted by Fetterman and Killeen (1990), is the experiment reported by Hicks and Allen (1979), in which subjects underestimated time in the explicit counting condition. This shows a possible influence of counting on the pacemaker. The present study also upholds such evidence, since the average productions (see Table 2) of the 3.6- and 4.8-sec interval conditions were generally significantly shorter in the conditions of counting - that is, when there were one, two, or three dividers-than in the no-segmentation (i.e., no-counting) condition.

Another fact related to the analysis of the coefficient of variation needs to be addressed. The coefficients in the present experiment were in general very low-the performances were good. The coefficients were generally somewhere around a value of .045 . These results are close to those reported by Fetterman and Killeen (1990; around .032 in their Experiment 3 ) and reported by others who have used a tapping task (.044: Ivry \& Keele, 1989; see also Ivry \& Corcos, in press). On the other hand, Wearden (1991a) reported a very much higher coefficient of variation (over .1).

Of theoretical interest are the results showing that the coefficient of variation was smaller at $4.8 \mathrm{sec}$ with three dividers than at $1.2 \mathrm{sec}$ with no divider. This may well be explained on the basis of the two-process model reported by Wing and Kristofferson (1973). They reported that the variance in the timing of a series of motor responses was due both to the variance of a timekeeper mechanism and to the variance in motor response delays related to, for example, neuromuscular transmission lags or movement time. The reduction of variability with a 4.8sec production would come from a reduction in the number of interventions of the motor system. More specifically, in a comparison involving the production of a 1.2sec production without segmentation versus the production of a 4.8-sec interval internally divided by four, each $1.2 \mathrm{sec}$ block is accompanied in the first case by the motor process variance, whereas in the second case, the motor activity occurred only once every four blocks. Consequently, the effect of the motor component variance was more emphasized with the 1.2-sec production. Indeed, this finding means that the production of a tap with the motor system causes more variance than does simply keeping 
active an internal count corresponding to this tap. Thus, the segmented input strategy might provide an interesting tool for estimating the cost, in variability, associated with the motor component of tapping. Finally, reintroduced in the context of Killeen and Weiss's (1987) model, this motor source of variability would be referred to as a constant error, $\alpha_{0}$.

As noted by Wearden (1991a), the study of chronometric counting was mysteriously neglected for many decades, until recent years. Interest in this question was mainly stimulated by the general theoretical framework developed by Killeen and Weiss (1987) that can account especially for the usefulness of the counting strategy. The results of the present experiment were integrated into this framework by addressing the question of optimal timing. The results also addressed the question of the properties of the information provided by the pacemaker. Indeed, although the present findings bring further support to the scalar rather than to the Poisson property, the exact nature of time information remains a fascinating question. Interesting attempts to describe the properties of time information - that is, the product of a pacemaker-are reported in the recent literature. Some researchers have addressed the question of properties, scalar or Poisson, of timing (Allan \& Gibbon, 1991; Fetterman \& Killeen, 1991; Gibbon, 1991; Killeen \& Fetterman, 1988; Wearden, 1991a); others have investigated the continuous versus the discrete nature of time (Killeen, 1992; Kristofferson, 1980, 1990); still others have proposed an interpretation of the pacemaker in terms of an oscillator (Treisman, Faulkner, Naish, \& Brogan, 1990) or oscillators (Church \& Broadbent, 1990; Miall, 1989). Finally, there have also been recent impressive demonstrations within the attempt to specify a locus for this clock component-namely, the cerebellum (Ivry \& Corcos, in press; Ivry \& Keele, 1989; Ivry, Keele, \& Diener, 1988). On the other hand, some research has rather been focused on how some factors, external or internal, could affect the activity of this pacemaker (Fetterman \& Killeen, 1991; Meck, 1991) and on the possibility of having a Poisson clock, but with scalar timing (Gibbon, 1991; Fetterman \& Killeen, 1990). All this recent activity, coming from mathematical, biological, and behavioral analyses, is making the study of time perception a strong and active field that should fulfill the hopes of Gibbon (1991) that an exciting analysis of cognitive mechanisms in the biology of time will be developed.

\section{REFERENCES}

Allan, L. G., \&ibron, J. (1991). Human bisection at the geometric mean. Leaming \& motivation, 22, 39-58.

Church, R. M., Broadbent, H. A. (1990). Alternative representations of time, number and rate. Cognition, 37, 55-82.

Creelman, D. C. (1962). Human discrimination of auditory duration. Journal of the Acoustical Society of America, 34, 582-593.

Fetterman, J. G., Killeen, P. R. (1990). A componential analysis of pacemaker-counter timing systems. Joumal of Experimental Psychology: Human Perception \& Performance, 16, 766-780.

Fetterman, J. G., Killeen, P. R. (1991). Adjusting the pacemaker. Learming \& Motivation, 22, 226-252.
Fetterman, J. G., \& Killeen, P. R. (1992). Time discrimination in Columba livia and Homo sapiens. Journal of Experimental Psychology: Animal Behavior Processes, 18, 80-94.

GETTY, D. J. (1975). Discrimination of short temporal intervals: A comparison of two models. Perception \& Psychophysics, 18, 1-8.

GetTy, D. J. (1976). Counting processes in human timing. Perception \& Psychophysics, 20, 191-197.

Gibbon, J. (1991). Origins of scalar timing. Leaming \& Motivation, 22, 3-38.

Gibbon, J., \& Church, R. M. (1984). Sources of variance in an information processing theory of timing. In H. L. Roitblat, T. G. Bever, \& H. S. Terrace (Eds.), Animal Cognition (pp. 465-488). Hillsdale, NJ: Erlbaum.

Gilliland, A. R., \& Martin, R. (1940). Some factors in estimating short time intervals. Joumal of Experimental Psychology, 27, 243-255.

Hicks, R. E., \& AlleN, D. A. (1979). Counting eliminates the repetition effect in judgments of temporal duration. Acta Psychologica, 43, 361-366.

IvRY, R. B., \& Corcos, D. (in press). Slicing the variability pie: Component analysis of coordination and motor dysfunction. In K. Newell \& D. Corcos (Eds.), Variability issues in motor control.

IVRY, R. B., \& KEELE, S. W. (1989). Timing functions of the cerebellum. Joumal of Cognitive Neuroscience, 1, 136-152.

IVRY, R. B., KeELE, S. W., Diener, H. C. (1988). Dissociation of the lateral and the medial cerebellum in movement execution. $E x$ perimental Brain Research, 73, 167-180.

KILleEN, P. R. (1992). Counting the minutes. In F. Macar, V. Pouthas, \& W. Friedman (Eds.), Time, action and cognition: Towards bridging the gap (pp. 203-214). Dordrecht: Klüwer.

KilleEn, P. R., \& Fetterman, J. G. (1988). A behavioral theory of timing. Psychological Review, 95, 274-295.

KILleEN, P. R., WeISS, N. A. (1987). Optimal timing and the Weber function. Psychological Review, 94, 455-468.

Kristofferson, A. B. (1977). A real-time criterion theory of duration discrimination. Perception \& Psychophysics, 21, 105-117.

Kristofferson, A. B. (1980). A quantal step function in duration discrimination. Perception \& Psychophysics, 27, 300-306.

Kristofferson, A. B. (1990). Timing mechanisms and the threshold for duration. In H.-G. Geissler (Ed.). Psychophysical explorations of mental structures (pp. 268-277). Toronto: Hogrefe \& Huber.

MECK, W. H. (1991). Modality-specific circadian rhythmicities influence mechanisms of attention and memory for interval timing. Leaming \& Motivation, 22, 153-179.

MiAlL, C. (1989). The storage of time intervals using oscillating neurons. Neural Computation, 1, 359-371.

Petrusic, W. M. (1984). Explicit counting and time-order errors in duration discrimination. In J. Gibbon \& L. Allan (Eds.), Timing and time perception (Annals of the New York Academy of Sciences: Vol. 423, pp. 630-633). New York: New York Academy of Sciences.

Rousseau, R., Picard, D., Pitre, E. (1984). An adaptive counter model for time estimation. In J. Gibbon \& L. Allan (Eds.), Timing and time perception (Annals of the New York Academy of Sciences: Vol. 423, pp. 639-642). New York: New York Academy of Sciences.

Treisman, M. (1963). Temporal discrimination and the indifference interval: Implications for a model of the "internal clock." Psychological Monographs, 77(Whole No. 576).

Treisman, M., Faulkner, A., Naish, P. L. N., Brogan, D. (1990). The internal clock: Evidence for a temporal oscillator underlying time perception with some estimates of its characteristic frequency. Perception, 19, 705-743.

WEARDEN, J. H. (1991a). Do humans possess an internal clock with scalar timing properties? Learning \& Motivation, 22, 59-83.

WEARDEN, J. H. (1991b). Human performance on an analogue of an interval bisection task. Quarterly Journal of Experimental Psychology, 43B, 59-81.

Wing, A. M., \& Kristofferson, A. B. (1973). Response delays and the timing of discrete motor responses. Perception \& Psychophysics, 14, 5-12.

(Manuscript received January 22, 1991 ; revision accepted for publication February 25, 1992.) 